\title{
A trip down memory lane with Retrovirology
}

\author{
Monsef Benkirane ${ }^{1}$, Ben Berkhout ${ }^{2}$, Persephone Borrow ${ }^{3}$, Ariberto Fassati ${ }^{4}$, Masahiro Fujii ${ }^{5}$, \\ J. Victor Garcia-Martinez ${ }^{6}$, D. Margolis ${ }^{6}$, Monique Nijhuis ${ }^{7}$, Leslie Parent ${ }^{8}$, Klaus Strebel $^{9}$, François Venter ${ }^{10}$, \\ Frank Kirchhoff ${ }^{11}$, Andrew Lever ${ }^{12^{*}}$, Susan Ross ${ }^{13^{*}}$ and Johnson Mak ${ }^{14^{*}}$
}

Fifteen years ago, Retrovirology was amongst the first open-access journals to be established through Biomed Central, instigated by our late Founding Editor Dr. KuanTeh Jeang. Since then, in what seemed like a rather daring move to be paper-free, Retrovirology has witnessed the exponential growth of open access journals that have changed the landscape of scientific publishing and communications. As was pointed out by the staff editors in PLoS Biology [1], the infancy of open access journal was a very different time from our present day, which was before smartphones, prior to most of the digital social media platforms and at a time when we had only begun to learn about the completed DNA sequences from the Human Genome Project [1].

Dr. Kuan-Teh Jeang (known universally as Teh) was an accomplished virologist and chief of the molecular virology section of the Laboratory of Molecular Microbiology at the NIAID NIH intramural program. Teh was a prolific scientist who authored more than 300 publications before he left us at the tragically young age of 54 . Through open access publishing, Teh saw an opportunity to create a platform for retrovirologists to publish their findings in a timely manner as well as actively disseminating these publications to the community. Many of us vividly remember the enthusiastic emails that Teh sent us regarding any news or hot-off the press publications from Retrovirology.

From the inaugural digital issue of Retrovirology, Teh pledged to serve our community by providing fast, fair, and responsive editing to all submissions [2], a

\footnotetext{
*Correspondence: amll1@medschl.cam.ac.uk; srross@uic.edu; j.mak@griffith.edu.au

${ }^{12}$ University of Cambridge, Cambridge, UK

${ }^{13}$ University of Illinois at Chicago, Chicago, USA

${ }^{14}$ Institute for Glycomics, Griffith University, Gold Coast, Australia

Full list of author information is available at the end of the article
}

philosophy that remains as the cornerstone of our practice in publishing with Retrovirology. Since day one, Teh amassed together an impressive team of Founding Associate Editors to lead this journal, including Mark Wainberg, Andrew Lever, Monsef Benkirane, Ben Berkhout, Masahiro Fujii, and Michael Lairmore. Throughout the years, a number of well-regarded researchers have also joined the team of Associate Editors and Guest Editors in Retrovirology, including Robin Weiss, Persephone Borrow, Ariberto Fassati, J. Victor Garcia-Martinez, Paul Gorry, David Margolis, Monique Nijhuis, Leslie Parent, Klaus Strebel, Francois Venter, Frank Kirchhoff, Marit van Gils, Alexander Pasternak, and Rogier Sanders.

In 2005, Teh garnered financial support to establish the Retrovirology Prize designed, not as a typical award for lifetime achievement to the most senior researchers, but aimed at mid-career scientists between the ages of 45 and 60-'arguably the most productive denizens of science' (as Teh might have put it) [3] or 'the glum-looking bunch of scientists' traversing their "midlife crises" (as Teh would have put it) [3]. Joking aside, the Retrovirology Prize has provided us with an opportunity to honour some of the amazing scientists who have contributed to our community at their 'mid-career' stages but who are not yet senior enough to receive the awards commonly given to reflect the end of a career. As one would expect, the Retrovirology Prize has since become a highly soughtafter award within our community, and many legends of our time are now part of this unique club of accomplished scientists (https://www.biomedcentral.com/collections/ retrovirology-prize). The annual deliberation process is often fierce but respectful, and the only constant being that there are more deserving individuals than the single award that we are able to give out each year. In 2005, the first recipient of Retrovirology Prize was Professor Stephen Goff at Columbia University [4], and recipients of 
subsequent Retrovirology Prize winners are listed (https ://www.biomedcentral.com/collections/retrovirologyprize). In recognition of the contribution of Teh to Retrovirology and to commemorate his untimely departure, the Retrovirology Prize was renamed as the Kuan-Teh Jeang Retrovirology Prize in 2015.

Throughout its short 15 years of existence, Retrovirology has grown from a modest journal that was largely driven by Teh, to a point where it is now recognised as a highly respected journal within our community. Retrovirology has witnessed and published a number of manuscripts that have had profound impacts on our field. While it is impractical to list or to recap all of the landmark works that have been published in Retrovirology, we have selected a few of our collative personal favourites to showcase some of the contributions that our colleagues have made through publications in Retrovirology.

Some of the most recognised publications include:

- Gila-Gaur and Strebel's review on HIV Vif and APOBEC proteins [5];

- Descours et al. research manuscript on SAMHD1 HIV restriction in quiescent $T$ cells [6];

- Sebastian and Luban's research report on Trim5a restricts retrovirus via capsid domain [7];

- Weiss's review on endogenous retroviruses [8];

- Towers's review on tripartite motif proteins and cyclophilin A [9].

Other timely manuscripts include:

- Work dissecting the relationship between HIV transcription and latency by van Lint et al. [10];

- The series of manuscripts that helped to show that xenotropic murine leukemia virus related virus (XMRV) is not a human pathogen [11-23];

- An early study that helped set the tone of using humanised mice to study HIV infection and pathogenesis in vivo by Berges et al. [24]; and

- The potential roles of microRNA in retrovirus related diseases [25-28].

Other publication highlights can also be found in the following weblinks (incorporating all of the papers that team of AEs suggest).

Retrovirology has done many things like this well, but there are still many areas for us to improve on including widening the diversity of our publications. Traditionally, Retrovirology was set up as a journal with a strong molecular virology focus, and we have not published a significant number of manuscripts that have immunological-, clinical or structural biological themes. As we look toward our future, Retrovirology will actively support manuscripts in these areas of retrovirus research that have not been well represented in our publication history.

We also plan to increase diversity in our editorial board, authors and readers. In an attempt to better reflect the diversity of the retrovirus community, our current editorial board comprises researchers from most of the retrovirus research-intensive countries. As we don't yet fully accurately reflect our community this is our aspiration and with the ongoing support from our submitting authors and readers, we are determined to make Retrovirology the journal that reflects the breadth of our collective values and commitment to our field.

With the sudden passing of Teh in 2013, Professors Mark Wainberg and Andrew Lever agreed to take on the roles of Co-Editor-in-Chief to lead our journal. Approaching the end of a 5 year term as Co-Editor-inChief for Retrovirology, Mark Wainberg sadly left us in an untimely manner in 2017 [29]. Mark had authored over 550 publication spanning his 30 years of work in retroviruses [29]. Andrew stepped down this year, becoming our first Editor emeritus. Retrovirology has survived all these ups and downs and with the support of the retroviral community we are beginning to see the metrics rise again.

With Andrew's retirement as Co-Editor-in-Chief from Retrovirology, we are delighted to have one of our original commissioned meeting reviewers in 2004 [30], Professor Susan Ross, takes the helm as Co-Editor-in-Chief with Johnson Mak to serve our research community. We have also added 4 social media editors (Felipe DiazGriffero, Robert Gifford, Sallie Permar, and Kei Sato) to the board, who will be publicizing findings of interest to the Retrovirology community.

We have also been proud to support the bi-annual Frontiers in Retrovirology Conference, which has brought together scientists from all over the world to discuss state-of-the-art findings in the field. The upcoming Fall 2020 conference in Prague (to be organized and led by Jiri Hejnar) promises to continue the tradition of outstanding scientific exchange in the field.

In ancient Greece at around 500 B.C., Heraclitus of Ephesus coined the phrase 'Change is the only constant', Retrovirology is no exception to this rule. To remain relevant and important to our loyal scientific contributors the journal must evolve and adapt so that it continues to provide a unique specialist forum for this fascinating branch of science and medicine.

\footnotetext{
Authors' contributions

JM, SR and AL drafted initial manuscript. All authors contributed to refinement of manuscript. All authors read and approved the final manuscript.
}

\section{Competing interests}

The authors declare that they have no competing interests. 


\begin{abstract}
Author details
${ }^{1}$ Centre National de la Recherche Scientifique, Montpellier, France. ${ }^{2}$ University of Amsterdam, Amsterdam, The Netherlands. ${ }^{3}$ University of Oxford, Oxford, UK. ${ }^{4}$ University College London, London, UK. ${ }^{5}$ Institute of Medicine and Dentistry, Niigata University, Niigata, Japan. ${ }^{6}$ University of North Carolina, Chapel Hill, USA. ${ }^{7}$ University Medical Center Utrecht, Utrecht, The Netherlands. ${ }^{8}$ Pennsylvania State University, Hershey, USA. ${ }^{9}$ NIAID, National Institutes of Health, Bethesda, USA. ${ }^{10}$ Wits Reproductive Health and HIV Institute, University of the Witwatersrand, Johannesburg, South Africa. ${ }^{11}$ University of Ulm, Ulm, Germany. ${ }^{12}$ University of Cambridge, Cambridge, UK. ${ }^{13}$ University of Illinois at Chicago, Chicago, USA. ${ }^{14}$ Institute for Glycomics, Griffith University, Gold Coast, Australia.
\end{abstract}

Published online: 21 August 2019

\section{References}

1. Editors, T. P. B. S. Fifteen years in, what next for PLOS Biology? PLoS Biol. 2018;16:e3000049. https://doi.org/10.1371/journal.pbio.3000049.

2. Jeang KT. Retrovirology and young Turks. Retrovirology. 2004;1:1. https:// doi.org/10.1186/1742-4690-1-1.

3. Jeang KT. Life after 45 and before 60: the Retrovirology Prize. Retrovirology. 2005;2:26. https://doi.org/10.1186/1742-4690-2-26.

4. Jeang KT. Small philanthropy and big science: the RETROVIROLOGY prize and Stephen P. Goff. Retrovirology. 2005;2:43. https://doi. org/10.1186/1742-4690-2-43.

5. Goila-Gaur R, Strebel K. HIV-1 Vif, APOBEC, and intrinsic immunity. Retrovirology. 2008;5:51. https://doi.org/10.1186/1742-4690-5-51.

6. Descours B, et al. SAMHD1 restricts HIV-1 reverse transcription in quiescent CD4(+) T-cells. Retrovirology. 2012;9:87. https://doi. org/10.1186/1742-4690-9-87.

7. Sebastian S, Luban J. TRIM5alpha selectively binds a restrictionsensitive retroviral capsid. Retrovirology. 2005;2:40. https://doi. org/10.1186/1742-4690-2-40.

8. Weiss RA. The discovery of endogenous retroviruses. Retrovirology. 2006;3:67. https://doi.org/10.1186/1742-4690-3-67.

9. Towers GJ. The control of viral infection by tripartite motif proteins and cyclophilin A. Retrovirology. 2007;4:40. https://doi. org/10.1186/1742-4690-4-40.

10. Van Lint C, Bouchat S, Marcello A. HIV-1 transcription and latency: an update. Retrovirology. 2013;10:67. https://doi. org/10.1186/1742-4690-10-67.

11. Groom HC, et al. Absence of xenotropic murine leukaemia virus-related virus in UK patients with chronic fatigue syndrome. Retrovirology. 2010;7:10. https://doi.org/10.1186/1742-4690-7-10.

12. Hohn $\mathrm{O}$, et al. Lack of evidence for xenotropic murine leukemia virusrelated virus(XMRV) in German prostate cancer patients. Retrovirology. 2009;6:92. https://doi.org/10.1186/1742-4690-6-92.

13. Hue $S$, et al. Disease-associated XMRV sequences are consistent with laboratory contamination. Retrovirology. 2010;7:111. https://doi. org/10.1186/1742-4690-7-111.

14. Smith RA. Contamination of clinical specimens with MLVencoding nucleic acids: implications for XMRV and other candidate human retroviruses. Retrovirology. 2010;7:112. https://doi. org/10.1186/1742-4690-7-112.
15. Sato E, Furuta RA, Miyazawa T. An endogenous murine leukemia viral genome contaminant in a commercial RT-PCR kit is amplified using standard primers for XMRV. Retrovirology. 2010;7:110. https://doi. org/10.1186/1742-4690-7-110.

16. Oakes $B$, et al. Contamination of human DNA samples with mouse DNA can lead to false detection of XMRV-like sequences. Retrovirology. 2010;7:109. https://doi.org/10.1186/1742-4690-7-109.

17. Robinson MJ, et al. Mouse DNA contamination in human tissue tested for XMRV. Retrovirology. 2010;7:108. https://doi. org/10.1186/1742-4690-7-108.

18. Smith RA, Gottlieb GS, Miller AD. Susceptibility of the human retrovirus XMRV to antiretroviral inhibitors. Retrovirology. 2010;7:70. https://doi. org/10.1186/1742-4690-7-70.

19. Qiu X, et al. Characterization of antibodies elicited by XMRV infection and development of immunoassays useful for epidemiologic studies. Retrovirology. 2010;7:68. https://doi.org/10.1186/1742-4690-7-68.

20. Jeziorski E, et al. No evidence for XMRV association in pediatric idiopathic diseases in France. Retrovirology. 2010;7:63. https://doi. org/10.1186/1742-4690-7-63.

21. Denner J. Detection of a gammaretrovirus, XMRV, in the human population: open questions and implications for xenotransplantation. Retrovirology. 2010;7:16. https://doi.org/10.1186/1742-4690-7-16.

22. Sakuma T, et al. No evidence of XMRV in prostate cancer cohorts in the Midwestern United States. Retrovirology. 2011;8:23. https://doi. org/10.1186/1742-4690-8-23.

23. Garson JA, Kellam P, Towers GJ. Analysis of XMRV integration sites from human prostate cancer tissues suggests PCR contamination rather than genuine human infection. Retrovirology. 2011:8:13. https://doi. org/10.1186/1742-4690-8-13.

24. Berges BK, Wheat WH, Palmer BE, Connick E, Akkina R. HIV-1 infection and CD4 T cell depletion in the humanized Rag2-/-gamma c-/- (RAG-hu) mouse model. Retrovirology. 2006;3:76. https://doi. org/10.1186/1742-4690-3-76

25. Ahluwalia JK, et al. Human cellular microRNA hsa-miR-29a interferes with viral nef protein expression and HIV-1 replication. Retrovirology. 2008;5:117. https://doi.org/10.1186/1742-4690-5-117.

26. Houzet $L$, et al. MicroRNA profile changes in human immunodeficiency virus type 1 (HIV-1) seropositive individuals. Retrovirology. 2008;5:118. https://doi.org/10.1186/1742-4690-5-118.

27. Yeung $M L$, et al. Changes in microRNA expression profiles in HIV1-transfected human cells. Retrovirology. 2005;2:81. https://doi. org/10.1186/1742-4690-2-81.

28. Scaria V, Hariharan M, Maiti S, Pillai B, Brahmachari SK. Host-virus interaction: a new role for microRNAs. Retrovirology. 2006;3:68. https://doi. org/10.1186/1742-4690-3-68.

29. Benkirane M, et al. Professor Mark Wainberg. Retrovirology. 2017;14:30. https://doi.org/10.1186/s12977-017-0353-6.

30. Freed EO, Ross SR. Retroviruses 2004: review of the 2004 Cold Spring Harbor Retroviruses Conference. Retrovirology. 2004;1:25. https://doi. org/10.1186/1742-4690-1-25.

\section{Publisher's Note}

Springer Nature remains neutral with regard to jurisdictional claims in published maps and institutional affiliations. 